\title{
Imaging Cytometry of Radiation-Induced Translocation of Nuclear HMGB1
}

\section{Linda Yasui}

\author{
Northern Illinois University, Dept. of Biological Sciences, DeKalb, IL 60115 USA
}

The ability of a protein to perform multiple roles in a cell is an adaptive strategy. One multi-functional, highly conserved protein is the non-histone, chromosomal architectural protein, high mobility group box 1 (HMGB1). In the cell nucleus, HMGB1 functions as a DNA chaperone by binding DNA and participating in regulation of gene expression and DNA replication, transcription, recombination and repair [1]. When HMGB1 is localized in the cell cytoplasm, HMGB1 promotes autophagy and facilitates inhibition of apoptosis when it binds beclin [2]. An extracellular localization of HMGB1 provides the context for HMGB1 to act as a danger signal promoting inflammation, immunity, autoimmunity, chemotaxis, cell proliferation and tissue regeneration by binding to RAGE and toll-like receptors [3]. Given that the subcellular location of HMGB1 provides different contexts for various functions of HMGB1, methods to assess the location of HMGB1 are essential to understand the various cellular roles of HMGB1. In this report, we present a method to assess the subcellular location of HMGB1 in human brain cancer cells in response to exposure to ionizing radiation.

To test the effect of ionizing radiation on translocation of HMGB1 in cells, human brain tumor, glioblastoma multiforme (GBM), U87 and U251 cells, were maintained in tissue culture and irradiated with 10 Gy $\gamma$ rays using a calibrated ${ }^{137} \mathrm{Cs}$ source as previously described [4]. At varying times after irradiation, cells were fixed and immunofluorescently stained to detect HMGB1. Stained cells were imaged using a Zeiss LSM 5 Pascal VarioTwo confocal laser scanning microscope as previously described [5].

ImageJ was used to determine the location of the fluoresecently stained HMGB1 molecule within a cell, revealing subcellular localization patterns and concentration of the molecule in subcellular compartments after exposure to ionizing radiation. Optical sections (stacks) through a cell were converted to a $\mathrm{Z}$ projection using ImageJ. The free-hand selection tool was used to select a region of interest within the $\mathrm{Z}$ projection image by outlining individual cells or individual cell nuclei. Image $\mathrm{J}$ calculated the area of selection (in pixels), the average gray value per pixel and the integrated density (sum of the pixel values in selected area or the product of Area and Mean Gray Value) of the selected area. A corrected total cell (or nuclear) fluorescence (CTCF or CTNF) value was calculated according to Burgess et al [6]. The corrected total cytoplasmic fluorescence (CTCytF) was obtained by subtracting the CTNF from the CTCF values for each treatment.

Imaging cytometry analysis reveals a radiation-induced translocation of HMGB1 in U87 and U251 cells (Table I). Since the whole cell and nuclear area changed dramatically with time after irradiation (especially in irradiated U251 cells), data for both cell lines were normalized by dividing corrected totals for whole cells or nuclei by the corresponding area of the cell or nucleus. Comparing normalized data, the CTCF value for irradiated U87 cells started decreasing at 18 hours and this trend continued for the cells imaged 3 days after IR. Correspondingly, the 18 hour and 3 day nuclear CTNF values also decreased, while CTcytF values varied in U87 cells.

Normalized CTCF values for U251 cells also decreased with increasing time after irradiation but the nuclear values slightly increased. 
In summary, the corrected total whole cell, nuclear and cytoplasmic values provide valuable data on movement of HMGB1 through the cell. The data suggest that HMGB1 in U87 is decreasing in the nucleus, while accumulating in the cytoplasm at around 18 hours after irradiation. By 3 days after irradiation, a lower fluorescent signal from HMGB1 indicates HMGB1 may be translocating to an extracellular compartment by that time. For U251 cells, the HMGB1 fluoresecent signal appears to peak at around 18 hours after IR but by 3 days after IR, HMGB1 appears to also start to translocate to the extracellular compartment, as was described for U87 cells. These data support a radiation-induced nuclear translocation of HMGB1 (Table I).

References:

[1] D Tang et al. Biochimica et Biophysica Acta (2010) 1799: 131-140.

[2] R Kang et al. Autophagy (2010) 6, 1-3.

[3] M Lotze et al., Immunological Reviews (2007) 220, 60-81.

[4] L Yasui, K Owens. International Journal of Radiation Biology (2012) 88, 980-990.

[5] LS Yasui. International Journal of Radiation Biology (2004) 80, 895-903.

[6] JA Burgess et al., Proceedings of the National Academy of Science, USA (2010) 107, 12564-12569.

[7] The author acknowledges funding from the Dept. of Defense, grant W81XH-10-1-017 and the technical assistance of Anne Wyer and Jay Highland.

TABLE I

Intensity or corrected total fluorescence (HMGB1) in the whole cell, nucleus or cytoplasm

U87 cells

\begin{tabular}{|l|l|l|l|l|}
\hline $\begin{array}{l}\text { A. } \\
\text { INTENSITY }\end{array}$ & $\mathbf{0}$ hrs post 0 Gy & $\mathbf{8}$ hrs post 10 Gy & $\mathbf{1 8}$ hrs post 10 Gy & $\mathbf{3}$ days post 10 Gy \\
\hline Whole cell & 55.15 & 50.26 & 47.13 & 35.46 \\
\hline Nucleus & 145.8 & 145.2 & 112.18 & 79.87 \\
\hline Cytoplasm & 24.0 & 19.36 & 24.66 & 17.61 \\
\hline
\end{tabular}

\section{U251 cells}

\begin{tabular}{|l|l|l|l|l|}
\hline $\begin{array}{l}\text { A. } \\
\text { INTENSITY }\end{array}$ & $\mathbf{0}$ hrs post 0 Gy & $\mathbf{8}$ hrs post 10 Gy & $\mathbf{1 8}$ hrs post 10 Gy & $\mathbf{3}$ days post 10 Gy \\
\hline Whole cell & 31.02 & 30.89 & 44.26 & 28.05 \\
\hline Nucleus & 97.26 & 112.28 & 152.51 & 119.73 \\
\hline Cytoplasm & 18.01 & 12.98 & 11.19 & 9.18 \\
\hline
\end{tabular}

